\title{
Approximate technique for calculation the celerity of long wave in channels with complex cross section
}

\author{
Sergei Sokolov ${ }^{1}$ (1)
}

Received: 5 June 2019 / Accepted: 9 January 2020 / Published online: 18 January 2020

(c) Springer Nature Switzerland AG 2020

\begin{abstract}
Dynamic processes in rivers are always associated with long waves of a different type. The celerity of the waves is main property for calculating their propagation, and the shape of channels cross section is one of the factors affecting the celerity. Previously, the approach based on the principles of the theory of energy motion was used to study the dynamics of the waves. Several analytical solutions for the celerity were obtained for the channels with simple geometry of cross section such as rectangular, triangular, and trapezoidal ones. Developing the approach for similar calculations in natural channels, which cross sections have complex shape, the approximate technique is proposed in the paper. The technique needs information on the geometric properties of the stream and channel. Obtained solutions were compared with some field measurements of tidal bores in natural channels. Aim of the paper is to further develop the approach, which is based on principles of energy motion, to the analysis of unsteady fluid flow.
\end{abstract}

Keywords Energy flux $\cdot$ Celerity of the wave $\cdot$ Long wave $\cdot$ Complex cross section $\cdot$ Tidal bore

\section{Introduction}

The technique proposed in the paper concerns the waves, which were defined by Rayleigh [6] as long waves. The typical examples are the flood waves, the waves after water release from reservoirs and also the dam-break waves. They are accompanied by water flow. Due to their long length, to describe the movement, they can be divided into successive elements having an individual celerity. Knowledge of the celerity of the wave phases and the wave front, above all, is important for mathematical modeling and prediction of their influence. It is believed that the theory of such waves is well-known for uniform channels of rectangular cross section. More complex cases when the channels have a different shape of cross section that affects the celerity of the waves are less studied. In addition, analysis and calculations require complex methods.
The influence of the shape of the channel cross section on the celerity of progressive waves was first discovered by Russell [8] in his experiments with solitary wave. In a flume with a rectangular cross section, the wave celerity $c$ corresponded to the equation

$c_{\text {rect }}=\sqrt{g\left(d_{0}+\eta\right)}$.

Here $g$ is the gravity acceleration; $d_{0}$ is the depth of water in repose, $\eta$ is the height of the crest of the wave above the plane of repose. Later, Rayleigh theoretically substantiated Eq. (1) in the referenced paper. In a flume with a triangular cross section, the measured wave celerity most closely corresponded to the equation

$c_{\text {triangle }}=\sqrt{\frac{1}{2} g\left(d_{0}+\eta\right)}$,

where $d_{0}$ is the depth of the flume in the middle.

Sergei Sokolov, sokolovsb@list.ru | ${ }^{1}$ Polytechnical Institute, Surgut State University, Lenin St., Surgut, Khanty Mansi Autonomous Okrug Yugra, Russia 628412. 
Perhaps, Rayleigh was the first to propose the equation for the celerity of a long wave in a channel with an arbitrary shape of cross section, which is

$c=\sqrt{g \frac{A_{0}}{B_{0}}}$,

where $A_{0}$ is the area of cross section below the undisturbed level, $B_{0}$ is the breadth at this level. The equation concerns only the celerity of the wave front, according to explanation. It does not take into account the dependence of the wave celerity on its height. Obviously, it is valid only for the case when $\eta \ll d_{0}$.

This paper continues the methods using principles of energy motion in fluids. The term "energy" concerns only the forms of mechanical energy, as is customary in hydromechanics.

In general, the technique discussed below is based on the theory of energy motion (TEM) [13]. The most important point of this theory is the understanding of energy as a "substance-like quantity" that is localized in a medium with a certain bulk density and moves as an energy flux. The principle of energy conservation in a unit volume of a medium is mathematically expressed as the continuity equation

$\frac{\partial e}{\partial t}+\operatorname{div}(\boldsymbol{q})=0$.

Here $e$ is the bulk density of energy, $t$ is the time, $\mathbf{q}=e \mathbf{c}$ is the vector of the energy flux density (Umov-Poynting vector), $\mathbf{c}$ is the vector of the velocity of energy motion. To date, TEM has had limited use, for example in acoustics to characterize the propagation of energy by sound waves.

The energy flux generated by the work of any force takes place in the form of so-called wave transfer. The velocity of corresponded energy transfer is equivalent to the celerity of wave propagation. In turn, the energy localized at any point in the medium provides the motion of the medium here. The intensity of the motion is determined by the density of energy.

Earlier, based on the principles of the theory, the process of energy propagation in a fluid and associated motion of the fluid itself were analyzed. The original energy analysis concerned the flow associated with long waves advancing in a horizontal channel with uniform cross section. Based on the analysis, analytical solutions were obtained for the case of rectangular and triangular cross section [11], which coincided exactly with experimental Eqs. (1) and (2). Thus, now we can assume that they are substantiated in terms of TEM. Next, the most general analytical solutions were obtained for the channels of prismatic (trapezoidal) and cylindrical types $[3$,
10]. They showed plausible relationship, despite the fact that they were not examined experimentally.

The analytical solutions were obtained for channels with different cross section but the shape was relatively simple. After this, the obvious question arises: are solutions possible for channels with complex or even arbitrary shape such as natural riverbeds? The solutions would spread to wider field of channel hydraulics and hydrology. Of course, the mathematical description of the cross section of such channels would be complex. This initially implies an error in the solutions, and a possible technique will be approximate.

The lack of experimental verification of the results limits the development of the approach. The description of experiments published in the literature rarely contains the necessary information. Therefore, one of the goals of the paper is to offer interested part of scientific community to examine the proposed technique.

The paper deals with the estimation of wave celerity only. It does not concern water flow velocity, though this important characteristic of streams, of course, is closely related with the character of long wave propagation.

\section{Methodology}

Since the approach is new, the analysis of energy and the associated fluid motion was limited to the simplest cases. A long wave generated in still water of constant depth was taken as an example from the book by Stoker [12]. A wave maker, which is a vertical plate, generates a water motion in an unlimited horizontal uniform hydraulic flume. Two main forms of the motion, which were called the simple wave and the depression wave, appear in the flume (Fig. 1a). Being variants of long wave, both types of the motion have the form of a flow in terms of kinematics. The flow with increasing level (simple wave) was mainly considered.

The external force that moves the wave maker with the velocity $V$ produces the work and transfers momentum and energy into water. Under accepted initial conditions, energy appearance in water is manifested both in motion and in a change of water level. Energy propagates along the flume as the energy flux $J$ (Fig. 1b).

The simplest example of the motion of a finite amount of energy is the solitary wave studied in detail by Russell. The volume of the localization of energy from the point of view TEM is shown in Fig. 2a where the motion of individual particles during the wave transmission is exhibited. The wave celerity $c$ is equivalent to that of energy.

Taking into account the peculiarity of the transfer of a single amount of energy, the energy flux can be represented as a continuous sequence of elementary amounts 

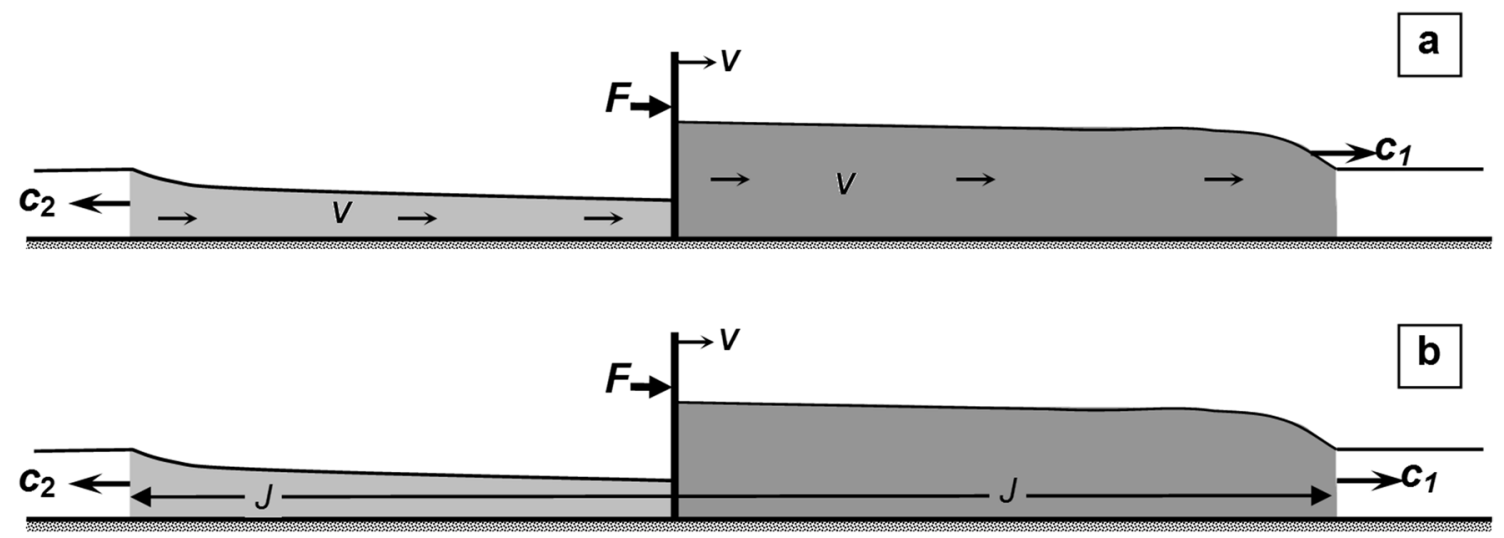

Fig. 1 Two basic forms of fluid flow in terms of energy propagation: a long waves induced by moving plate; $\mathbf{b}$ the waves in pictures of the energy flux. Here, $F$ is the external force; $J$ is the energy flux; $V$ is the velocity of water flow; $c_{1}, c_{2}$ is the celerity of the simple wave and depression wave (e.g., the celerity of energy propagation)
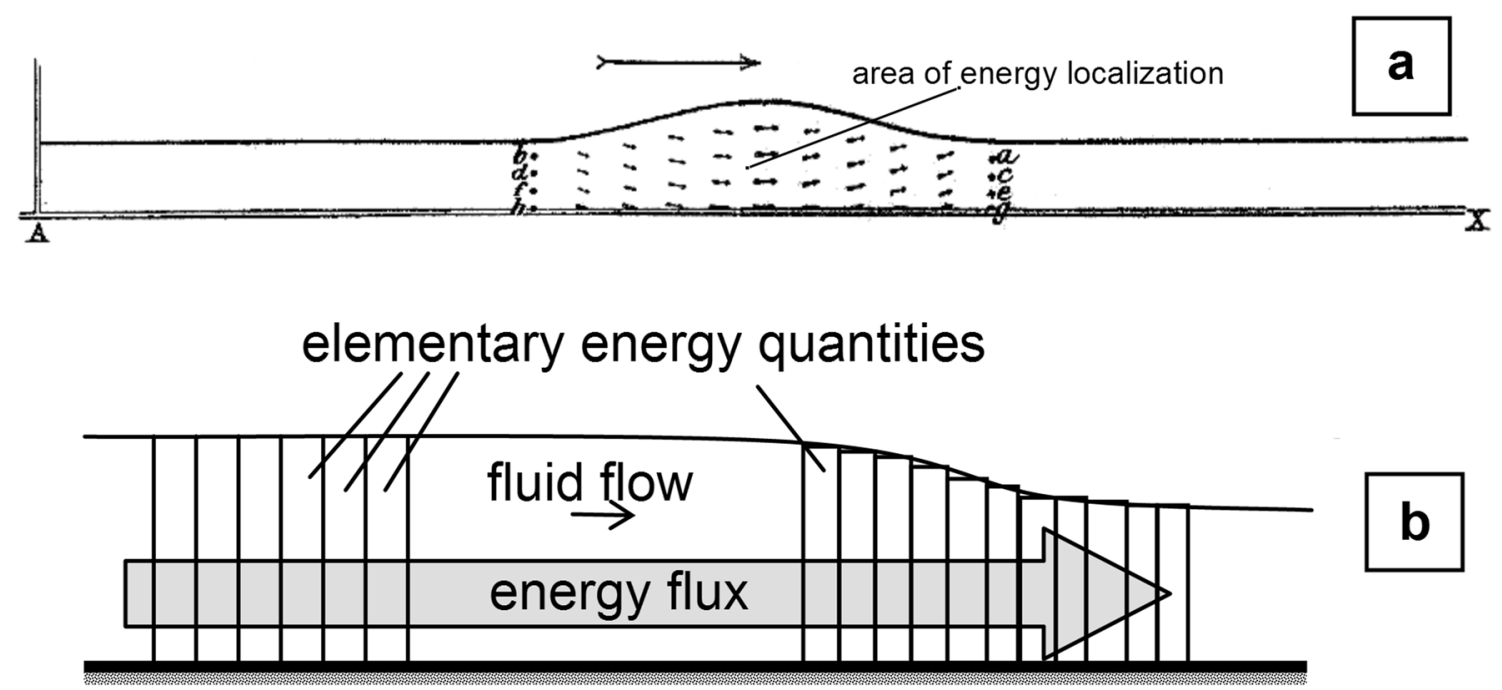

Fig. 2 Localization of energy in fluid during wave transmission: a in a solitary wave [8, Plate XVII, Fig. 5]; b in a long simple wave

(Fig. 2b). They are transported with a certain bulk density in the form of elementary waves. Thus, in order to describe the entire wave, it is important to know the energy density of each individual part, as well as their celerity.

According to earlier results (see, for instance, [11]), the celerity $c$ of long wave and each of its element in a channel are related with the value of energy transported with the wave. The relationship has the following view

$c^{2}=\frac{2}{\rho} \frac{e_{U}}{\left(1-\frac{A_{0}}{A}\right)^{2}}$.

Here $\rho$ is the density of fluid; $e_{U}$ is the density of potential energy in the area of running wave that is averaged over channel cross section; $A_{0}, A$ are the area of the cross section before and during the wave transmission, correspondingly.

Equation (5) was obtained under following considerations. While the wave forms in the flume, an increase in the energy of the fluid occurs at the surface of the wave maker in the form of all new wave elements. During the time $\mathrm{d} t$, a new wave element is formed, occupying the volume $\mathrm{d} W$. It contains the amount of energy $\mathrm{d} E$ with a finite density $e=\mathrm{d} E / \mathrm{d} W$. If the wave maker moves with a certain velocity $V$ at this time, the fluid in this volume has the same velocity due to boundary conditions. Since this is a new wave element, the change in the momentum of the fluid is $\rho V \cdot d W$. Then the force $F$, which acts at this moment on the fluid, can be written as $\rho V \cdot d W / d t$ according to Newton's second law. The work done will 
be equal $F V \cdot d t=\rho V^{2} \cdot d W$. The amount of energy received will be equivalent to the work and equal to

$\mathrm{d} E=\rho V^{2} \mathrm{~d} W$.

The mean density of energy in the volume $\mathrm{d} W$ is

$e=\rho V^{2}$.

Further behavior of this portion of energy is expected to be the same as in the case of an ordinary solitary wave; it moves along the channel, passing from one volume of fluid to another.

It is seen that the kinetic energy $\left(\rho V^{2} / 2\right) \cdot d W$ is only a half of total amount. Obviously, the second half is due to the potential energy of the deformation of the volume $\mathrm{d} W$. It means the densities of the potential energy $e_{\mathrm{K}}$ and kinetic energy $e_{K}$ are equaled

$e_{U}=e_{\mathrm{K}}$.

It follows from the condition of conservation of volume $\mathrm{d} W$, which was deformed by the wave maker during movement (see Fig. 3a)

$\frac{V}{C}=1-\frac{A_{0}}{A}$.

The deformation of the volumes in the flume (Fig. 3b) occurs similarly to the deformation near the wave maker. Therefore, all arguments and Eqs. (6)-(9) are valid for an arbitrary cross section of the flume. The solution of the system of Eqs. (6)-(9) gives Eq. (5). It expresses the wave celerity through the geometric characteristics of the flow, which are much easier to determine than the flow velocity, especially if the channel cross section has a simple geometric shape.

\section{Results and discussion}

The effect of channel cross section shape in Eq. (5) appears just both in different density of energy and in the ratio of area values $A_{0} / A$. The value of potential energy of water volumes deformation is easily determined in the channels, which cross section can be described mathematically. However, it is hard to do analytically for natural channels with irregular shape of that. Proposed solution is to use the density of energy estimated only for the volume of deformation instead of that for whole water volume. The volume of deformation is the volume of the wave disturbance that is above the undisturbed water level.

Consider the approach for the channel with rectangular cross section that is the simplest geometrically (Fig. 4). Here, the wave perturbation is shaded part of cross section above the undisturbed water level 0-0.

For instance, in a rectangular channel, the potential energy of deformation of any volume of water between two cross sections is determined by the following formula

$U=\rho g \frac{\left(d-d_{0}\right)^{2}}{2 d} W$.

Here $d$ is the full depth in the area of the wave. The equation was obtained as the work produced by the force of hydrostatic pressure in the fluid volume $W$, while it returns from disturbed state with the depth $d$ to the initial state of equilibrium with the depth $d_{0}$. Mean density of energy for Eq. (5) is

$e_{U}=\frac{U}{W}=\rho g \frac{\left(d-d_{0}\right)^{2}}{2 d}$.

Analytical solutions for $U$ and $e_{U}$ in non-rectangular channels differ from Eqs. (10) and (11) and have more complex expression.
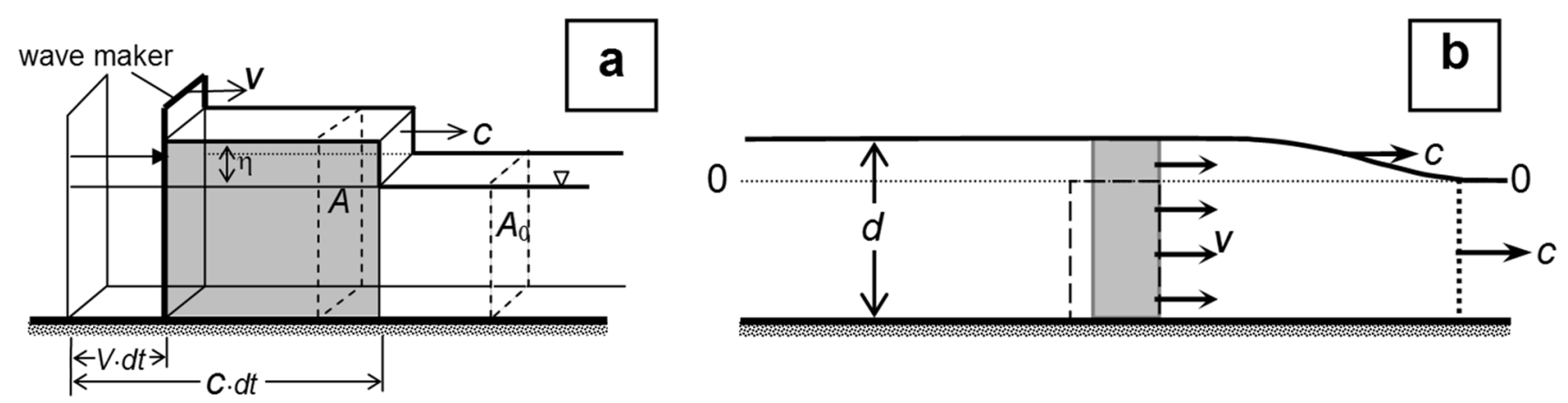

Fig. 3 Deformation of a water volume in the wave of elevation: $\mathbf{a}$ at the moment of wave formation near wave maker; $\mathbf{b}$ deformation of water volume in the flume during the wave transmission. The deformed volume is shaded 

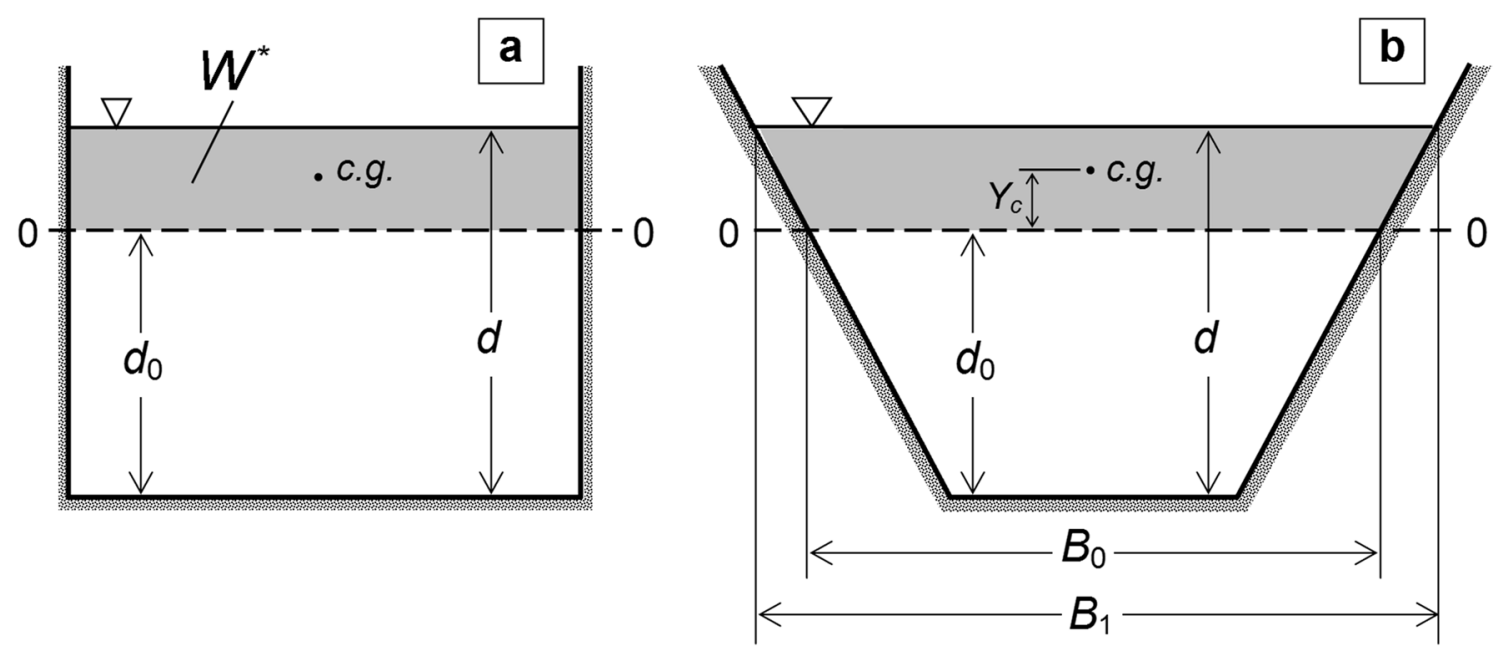

Fig. 4 Definition sketch of channel cross section during transmission of the wave of elevation: $\mathbf{a}$ in a rectangular channel; $\mathbf{b}$ in a trapezoidal channel. The area of the wave perturbation is shaded; c.g. is the center of gravity of wave perturbation

What about rectangular channels, the same value of potential energy given by Eq. (10) can also be expressed differently

$U=\rho g \frac{d-d_{0}}{2} W^{*}$.

Here $W^{*}$ is the volume of the wave perturbation above the level 0-0 (see Fig. 4).

As the value of potential energy in Eq. (12) is proportional to the volume of fluid as it is easy to express the bulk density as

$e_{U}^{*}=\frac{U}{W^{*}}=\rho g \frac{d-d_{0}}{2}$,

However, $e^{*}$ is overvalued because it is estimated with respect to the volume $W^{*}$ that is smaller than in Eq. (10). The values of the density of energy obtained by Eqs. (11) and (13) are inversely proportional to corresponded volumes. Thus, the relationship between the values is

$e_{U}=e_{U}^{*} \frac{W^{*}}{W}$

For the channels with uniform cross section or for two close sections located at the small distance $\Delta L$ between them, the volume $W$ is written as $A \cdot \Delta L$, while $W^{*}$ is written as $\left(A-A_{0}\right) \cdot \Delta L$. Their ratio in Eq. (14) is

$\frac{W^{*}}{W}=1-\frac{A_{0}}{A}$.

Replacing $e_{U}$ in Eq. (5) with its expression in Eq. (14) and using Eq. (15), we obtain the analogue of Eq. (5) as $c^{2}=\frac{2}{\rho} \frac{e_{U}^{*}}{\left(1-\frac{A_{0}}{A}\right)}$.

For the channels with rectangular cross section, the density of energy $e_{U}^{*}$ in Eq. (13) is the function of $\left(d-d_{0}\right) / 2$ that is the height of the center of gravity $Y_{C}$ of the volume $W^{*}$ above the undisturbed water level 0-0

$\frac{d-d_{0}}{2}=Y_{C}$.

Let us to assume for non-rectangular channels, the density of energy $e_{U}^{*}$ of wave perturbation is also related with the height of its center of gravity by following general equation

$e_{U}^{*}=\rho g Y_{\mathrm{C}}$.

Then, the circumstance gives new opportunities for estimation of long wave celerity.

Value of $Y_{C}$ can be calculated with well-accuracy if the height of the wave is known as well as the shape of channel cross section. In any rectangular channel (Fig. 4a), for instance, the cross section of wave perturbation is also rectangular and the value of $Y_{C}$ has to be calculated by Eq. (17). Using it in Eq. (16), we obtain the solution for the wave celerity as Russell's Eq. (1). If channel cross section is trapezoidal or triangular, then the shape of wave part of the cross section is trapezoidal (Fig. 4b). If $\Delta d=d-d_{0}$, the value of $Y_{C}$ can be expressed as 
$Y_{\mathrm{c}}=\frac{\Delta d}{3} \frac{B_{0}+2 B_{1}}{B_{0}+B_{1}}$.

Here $B_{0}$ is the free surface width before wave transmission (at the level $0-0$ ); $B_{1}$ is the free surface width during wave transmission.

The approach was verified for the case of trapezoidal and triangular channels. Solutions coincided completely with those obtained earlier by particular formulae [3].

Being based on these arguments, the assumption made for Eqs. (16) and (18) looks plausible. Therefore, the same approach could be applied to estimate the celerity of the waves running in the channels with more complex shape of cross section like natural ones. To use the approach, it needs to calculate the height $Y_{C}$ of the part of river cross section which is located above undisturbed water level above the level. Necessary data of the shape and the area of channel cross section at different water levels can be obtained by depth measurements. Then, using Eqs. (18) and (16), the wave celerity can be expressed as

$c=\sqrt{\frac{2 g Y_{C}}{1-\frac{A_{0}}{A}}}$.

The shape of wave perturbation may be approximated as trapezoidal (Fig. 5) for the channels with complex cross section. Then, Eq. (19) is used to calculate $Y_{C}$.

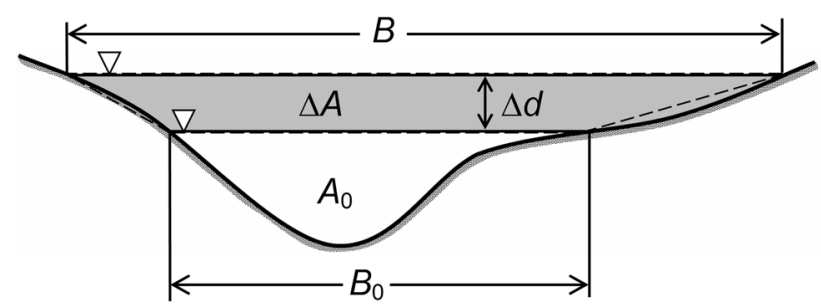

Fig. 5 Definition sketch of the wave of elevation (approximated wave perturbation area is contoured by dotted line)

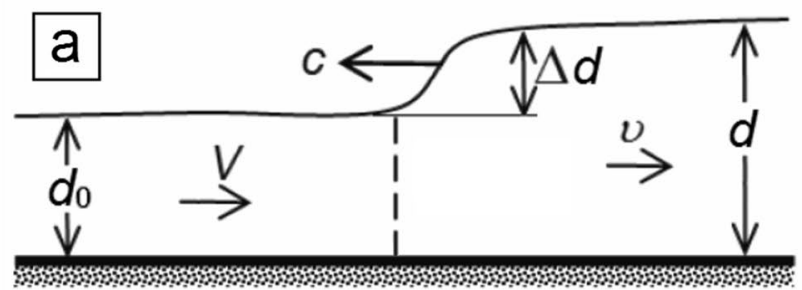

Fig. 6 Tidal bore and its interpretation in terms of energy fluxes: a definition sketch and kinematical structure: $V$ is the mean flow velocity in the stream before tidal bore passage (incident wave); $v$ is
The solution based on Eq. (20) was tested using tidal waves as an example. The choice was due to the fact that the necessary data concerning the long waves directly could not be found but the measurements made for tidal waves had all the data. Despite the fact that tidal waves usually do not belong to the category of long waves and they advance against the river stream (Fig. 6a), they look similar to the long waves in terms of energy flux. One of the possible cases of its origin is partial reflection of energy flux of river stream in estuary because of sea level rise. As a result, the incident energy flux $J_{i}$ of main river stream is divided into reflected $J_{r}$ and refracted (passed) ones. The reflected energy flux propagates upstream as tidal wave.

Incident and reflected fluxes exist simultaneously in the channel superposing each other. The reflection can be perfect and imperfect. In the case of perfect reflection, incident and reflected energy fluxes have equaled values and their momentums have opposite sign. Therefore, the flow in the channel in the area of tidal wave interrupts. In the case of imperfect reflection, the reflected energy and momentum fluxes are less than the incident ones. The flow does not interrupt, however, the mean velocity of the flow becomes less here being the result of superposition of two opposite fluxes with different values (Fig. 6b).

The flow velocity of the incident wave certainly effects on the flow velocity in the reflected one. However, its influence on the celerity of the wave itself is the question because the tidal waves as well as the directional long waves are not only the surface waves in terms of energy flux. The celerity depends only on the density of their energy flux as it was noted above. Assuming that the dependence is more complex, it was nevertheless assumed here that the river flow velocity that is the incident wave does not affect the celerity of the tidal wave and was not taken into account.

Data of field measurements of tidal bore celerity in river estuaries performed by different authors $[1,4,5,9,14]$ are used to examined the technique. They were collected by Chanson [2] and added significantly in [7]. All the data

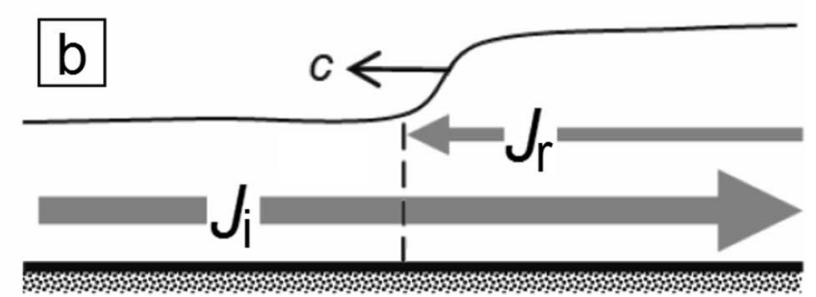

the mean flow velocity in the area of tidal bore $(V>u)$; $\mathbf{b}$ the energy fluxes: $J_{i}$ is forward (incident) one, $J_{r}$ is backward (reflected) one 
Table 1 Field measurements of tidal bore cross-sectional properties [7] and comparison of measured $c_{\mathrm{m}}$ and calculated $c_{\mathrm{c}}$ values of tidal bore celerity

\begin{tabular}{|c|c|c|c|c|c|c|c|c|c|c|c|}
\hline \multirow[t]{2}{*}{ References } & \multirow[t]{2}{*}{ River } & \multirow[t]{2}{*}{ Year } & \multicolumn{6}{|c|}{ Field measurements data } & \multicolumn{3}{|c|}{ Calculated data } \\
\hline & & & $B_{0}(\mathrm{~m})$ & $B_{1}(\mathrm{~m})$ & $\Delta d(\mathrm{~m})$ & $A_{0}\left(\mathrm{~m}^{2}\right)$ & $\Delta A\left(\mathrm{~m}^{2}\right)$ & $c_{m}(\mathrm{~m} / \mathrm{s})$ & $Y_{C}(m)$ & $A\left(\mathrm{~m}^{2}\right)$ & $c_{c}(\mathrm{~m} / \mathrm{s})$ \\
\hline [9] & Dee river & 2003 & 68.3 & 72.8 & 0.45 & 39.3 & 31.4 & 4.10 & 0.23 & 70.7 & 3.17 \\
\hline [14] & Daly river & 2003 & 129.2 & 130.9 & 0.28 & 289.3 & 36.4 & 4.70 & 0.14 & 319.7 & 4.94 \\
\hline \multirow[t]{2}{*}{ [5] } & Sélune river & 2010 & 34.7 & 116.9 & 0.34 & 5.25 & 27.3 & 2.00 & 0.20 & 32.55 & 2.17 \\
\hline & & & 33.2 & 117 & 0.41 & 3.56 & 31.3 & 1.96 & 0.24 & 34.86 & 2.30 \\
\hline \multirow[t]{2}{*}{ [1] } & Garonne river & 2010 & 75.4 & 81.6 & 0.50 & 105.7 & 39.4 & 4.49 & 0.25 & 145.1 & 4.28 \\
\hline & & & 75.8 & 81.6 & 0.46 & 108.8 & 36.0 & 4.20 & 0.23 & 144.8 & 4.28 \\
\hline \multirow[t]{10}{*}{ [7] } & Garonne river & 2012 & 79.0 & 84.3 & 0.45 & 158.9 & 36.71 & 3.85 & 0.23 & 195.61 & 4.87 \\
\hline & & & 78.7 & 84.3 & 0.52 & 152.3 & 42.24 & 4.58 & 0.26 & 194.54 & 4.87 \\
\hline & & 2013 & 65.0 & 67.0 & 0.30 & 85.6 & 19.80 & 4.32 & 0.15 & 105.40 & 3.97 \\
\hline & & 2015 & 67.6 & 69.9 & 0.338 & 101.4 & 23.24 & 4.23 & 0.17 & 124.64 & 4.23 \\
\hline & & & 64.3 & 67.4 & 0.470 & 72.8 & 30.72 & 4.25 & 0.24 & 103.52 & 3.96 \\
\hline & & & 65.1 & 69.5 & 0.496 & 56.6 & 33.30 & 4.79 & 0.25 & 89.90 & 3.64 \\
\hline & & & 64.5 & 67.6 & 0.44 & 74.9 & 29.07 & 4.45 & 0.22 & 103.97 & 3.94 \\
\hline & & & 65.9 & 69.1 & 0.48 & 88.0 & 32.23 & 4.61 & 0.24 & 120.23 & 4.21 \\
\hline & Garonne river & 2016 & 70.3 & 74.0 & 0.50 & 118.1 & 37.79 & 4.26 & 0.25 & 155.89 & 4.52 \\
\hline & & & 71.5 & 73.5 & 0.33 & 122.3 & 23.70 & 4.44 & 0.17 & 146.00 & 4.47 \\
\hline [4] & Sée river & 2012 & 21.7 & 23.9 & 0.56 & 14.82 & 12.90 & 3.20 & 0.28 & 27.72 & 3.46 \\
\hline
\end{tabular}

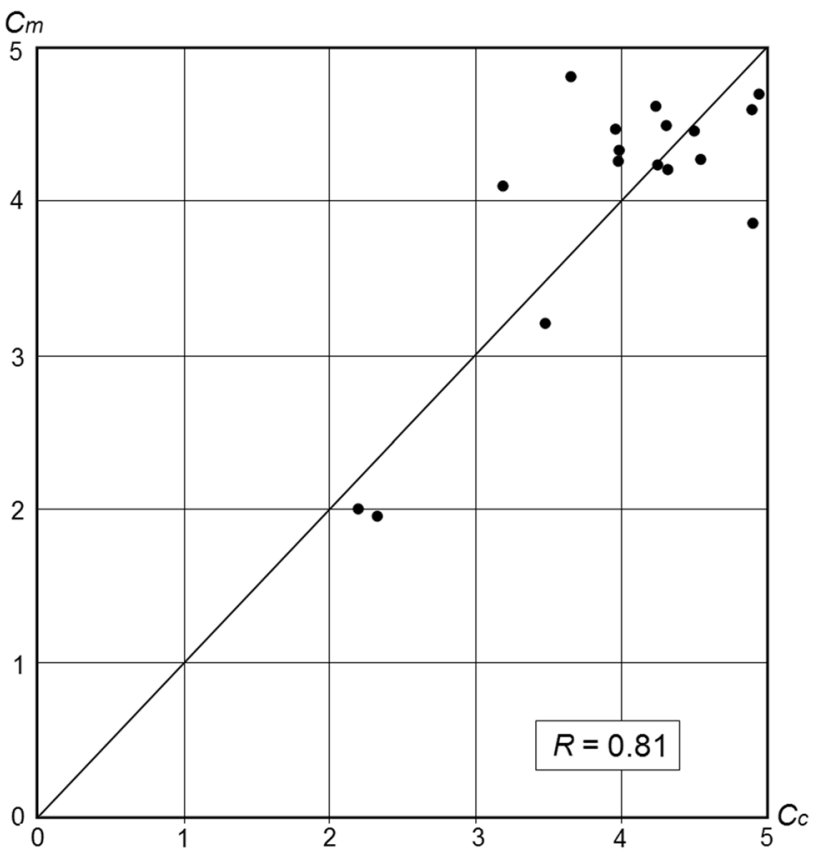

Fig. 7 Relationship of the measured values of the tidal wave celerity $\mathrm{Cm}$ versus calculated ones $\mathrm{Cc}$

necessary for calculation (see Table) were selected from noted papers (Table 1).

Using the technique, the height of the center of gravity $Y_{C}$ of the wave part of river cross section was calculated. Its shape was assumed like reverse trapezoid or close as it is shown in Fig. 5. The value of $Y_{C}$ was calculated by Eq. (19). Final results are given in the table. Comparison of measured and calculated data is shown in Fig. 7.

\section{Conclusion}

Despite the fact that the comparison values of tide celerity $c_{c}$ calculated according to Eq. (20), and the measured values $c_{m}$ seems not very good graphically, the coefficient of correlation is rather high $(R>0.8)$. Of course, it should be borne in mind that field measurements have limited accuracy, which is lower than in laboratory experiment. On the one hand, high correlation gives hope that this approximate technique has the prospects of practical application. On the other hand, the value is determined largely due to the data of measurements made on Sélune River [5] for this dataset. Obviously, the dataset must have a wider range to make a more reliable conclusion. It seems, only laboratory experiments with given range of conditions and providing accurate measurements can give such additional information. Verification of the propagation of directional waves would be preferable.

Acknowledgements The paper is the first attempt to verify the theoretical approach that is based on the principles of energy motion using independent measurements. Author express his appreciation to professor H.Chanson (The University of Queensland) for the data 
of field measurements of tidal bore those permitted to compare the results.

\section{Compliance with ethical standards}

Conflict of interest The authors declare that they have no conflict of interest.

\section{References}

1. Chanson H, Reungoat D, Simon B, Lubin P (2011) High-frequency turbulence and suspended sediment concentration measurements in the Garonne River tidal bore. Estuarine Coastal Shelf Sci 95(2-3):298-306

2. Chanson $\mathrm{H}$ (2012) Momentum consideration in hydraulic jumps and bores. J Irrig Drain Eng 138(4):382-385

3. Egorov EE, Sokolov SB (2016) Elevation wave velocity in a trapezoidal channel. Power Technol Eng 50(4):361-364. https://doi. org/10.1007/s10749-016-0713-8

4. Furgerot L, Mouaze D, Tessier B, Perez L, Haquin S (2013). Suspended sediment concentration in relation to the passage of a tidal bore (Sée River Estuary, Mont Saint Michel, NW France). In: Proc. Coastal Dynamics 2013, Arcachon, France. pp. 671-682

5. Mouaze D, Chanson H, Simon B (2010) Field measurements in the tidal bore of the Sélune River in the Bay of Mont Saint Michel. Hydraulic Model Rep. No. CH81/10, School of Civil Engineering, Univ. of Queensland. Brisbane, Australia

6. Rayleigh J (1876) On waves. Philos. Mag. 4(1):257-279
7. Reungoat $D$, Lubin P, Leng $X$, Chanson H (2018) Tidal bore hydrodynamic and sediment processes: 2010-2016 field observation in France. Coast Eng J. https://doi.org/10.1080/21664 250.2018.1529265

8. Russell JSC (1845) Report on waves. Report on the 14th meeting British Assoc. for the Advancement of Science. John Murray, London, pp. 311-390

9. Simpson JH, Fisher NR, Wiles P (2004) Reynolds stress and TKE production in an estuary with a tidal bore. Estuarine Coast Shelf Sci 60(4):619-627. https://doi.org/10.1016/s0272 $-7714(04) 00065-4$

10. Sokolov SB (2003) On the estimation of long waves velocity in the channels with parabolic cross-section. Water Manag Rus 2:135-151 (in Russian)

11. Sokolov S (2007) Speed of long wave in a channel with triangular cross section. In: Proc. of 32nd IAHR World Congress, Venice. Italy. [CD-ROM]

12. Stoker JJ (1957) Water waves. The mathematical theory with applications. Interscience Publ., New York

13. Umow N (1874) Ableitung der bewegungsgleichungen der energie in continuirlichen körpern. Zeitschrift fur Mathematik und Physik 19:418-431 (in German)

14. Wolanski E, Williams D, Spagnol S, Chanson H (2004) Undular tidal bore dynamics in the Daly Estuary, Northern Australia. Estuarine Coast Shelf Sci 60(4):629-636

Publisher's Note Springer Nature remains neutral with regard to jurisdictional claims in published maps and institutional affiliations. 